\title{
El uso de la acupuntura en las alteraciones del sistema musculo-esquelético de los equinos
}

\section{The use of acupuncture in alterations of the musculoskeletal and nervous system of horses}

\author{
Bautista Villamil Yeisson Mauricio ${ }^{1}$ y Fuentes Reyes Edgar Edilberto ${ }^{2}$ \\ ${ }^{1} \mathrm{MVZ}$, Unillanos y ${ }^{2} \mathrm{MVZ}$, Esp., MSc, PhD, Docente Unillanos \\ efuentes@unillanos.edu.co
}

Recibido 24 de Enero de 2017, Aceptado 21 de Abril 2017

\section{RESUMEN}

La acupuntura es una técnica milenaria china que está siendo empleada dentro de la medicina veterinaria, la cual tiene dos corrientes: tradicional y transposicional, que es la empleada en la medicina occidental por su fácil aprendizaje de la localización de los acupuntos; ésta se basa en canales (meridianos) que recorren todo el cuerpo y por medio de estos hay un flujo de energía denominado Qi. La acupuntura se emplea para el tratamiento de diferentes patologías, enfocándose este artículo en las del sistema musculo-esquelético y nervioso; además de ayudar con la recuperación de los pacientes, el uso de la acupuntura en equinos mejora su desempeño especialmente aquellos que están sometidos a esfuerzo físico. El estímulo generado por cada punción en determinado acupunto, genera un efecto benéfico para el paciente, puesto que son áreas con una densidad alta en terminaciones nerviosas, mastocitos, vasos linfáticos, arteriolas y vénulas, liberando endorfinas post-estímulo, para generar el estímulo de los acupuntos hay varias técnicas, incluyendo la de aguja seca, electroacupuntura, moxibustión e implantación. La acupuntura se puede emplear en los equinos en patologías como: laminitis, claudicaciones y lumbalgias; y en condiciones nerviosas como: parálisis del nervio radial, síndrome de Wobbler y convulsiones; además mejora la calidad de vida de los animales tanto enfermos como sanos.

Palabras clave: Acupuntos, laminitis, claudicaciones, medicina alternativa. 


\section{ABSTRACT}

Acupuncture is a Chinese millennial technique that is being used within the Veterinary Medicine, which has two currents: traditional and transpositional, that is the used in Western medicine for its easy learning of the location of acupoints; this is based on channels (meridians) that run through the body and through these there is a flow of energy called Qi. Acupuncture is used for the treatment of different pathologies, focusing this article on those of the musculoskeletal and nervous system; en addition to helping with patient recovery, the use of acupuncture in horses improves their performance especially in those who are subjected to physical exertion. The stimulus generated by each puncture in a given acupoint, generates a beneficial effect for the patient, because they are areas with a high density of nerve endings, mast cells, lymph vessels, arterioles and venules, releasing post-stimulus endorphins; to generate the stimulation of acupoints there are several techniques, including the dry needle, electroacupuncture, moxibustion and implantation. The acupuncture can be used in horses in pathologies such as: lameness, claudications and low back pain; and in nervous conditions such as: radial nerve palsy, Wobbler syndrome and seizures; in addition improving the quality of life of both sick and healthy animals.

Keywords: Acupoints, lameness, claudications, alternative medicine.

\section{RESUMO}

A acupuntura é uma técnica chinesa antiga que está sendo usada dentro da medicina veterinária, a qual tem duas correntes: tradicional e transposicionais, que é usado na medicina ocidental por sua fácil aprendizagem da localização dos pontos de acupuntura; é baseado em canais (meridianos) que correm por todo o corpo e, através destes, há um fluxo de energia chamada Qi. Acupuntura é utilizada para o tratamento de doenças diferentes, concentrando-se este artigo no músculo-esquelético e do sistema nervoso; além de ajudar com a recuperação dos pacientes, o uso da acupuntura em cavalos melhora o desempenho especialmente aqueles que estão submetidos a esforço físico. O estímulo gerado por cada 
punção em determinado acupontos gera um efeito benéfico para $\circ$ paciente, porque são áreas com uma alta densidade de terminações nervosas, mastócitos, vasos linfáticos, arteríolas e vénulas, libertando endorfinas pós-estímulo; para gerar o estímulo dos acupontos existem várias técnicas, incluindo a de agulha seca, eletroacupuntura, moxabustão e implementação. $O$ acupuntura pode ser usada em patologias eqüinos como: laminites, claudicação e lumbago; e condições do sistema nervoso tais como: paralisia do nervo radial, síndrome de Wobbler e convulsões; além melhora a qualidade de vida dos animais tanto doentes e saudáveis.

Palavras-chave: Acupontos, laminites, claudicação, medicina alternativa.

\section{INTRODUCCIÓN}

La acupuntura (AP) se basa en la inserción de agujas en puntos específicos del cuerpo para producir una reacción fisiológica generando una acción analgésica, un efecto antiespasmódico, descongestionante y aumento en la capacidad de resistencia del organismo a diversas agresiones a las cuales está sometido (Silva et al., 2008). La AP nació en el extremo oriente, en China, según datos arqueológicos alrededor de hace unos 5000 años (Scognamillo y Becharall, 2010); llego a occidente en el siglo XVII, según informes realizados por los jesuitas franceses que se encontraban en Pekín, los cuales fueron publicados en Francia en 1671 y 1682 (Sussmann, 2004). La AP en equinos data de hace 2000-3000 años A.C., durante la dinastía Shang y Chow en china; alrededor del año 650 A.C. Bai-le escribió "Bai- le's Canon of Veterinary Medicine" uno de los primeros textos de medicina veterinaria, el cual fue primordialmente sobre acupuntura $y$ moxibustión enfatizado en la medicina equina (Schoen, 2000).

La AP es un tratamiento no invasivo, de fácil aplicación, no requiere de instalaciones e instrumentales complejos, y es de bajo costo, además de ser indicado en enfermedades que cursan con dolor agudo o crónico debido a que las punciones producen intensos efectos analgésicos (Foganholli et al., 2007), ha sido usada exitosamente en el tratamiento de varias condiciones musculo-esqueléticas 
como los problemas de corvejón, laminitis, enfermedad navicular, discoespondilitis; y nerviosas como parálisis de los nervios facial, ciático y radial, convulsiones, paraparesias, paraplejias, y accidentes cerebrovasculares en los equinos, ya sea como tratamiento primario o como coadyuvante en las terapias veterinarias convencionales (Foganholli y Filadelpho, 2007; Schoen, 2000), mediante el empleo de los acupuntos de la AP transposicional, que son los más utilizados por médicos occidentales por su fácil aprendizaje, usando diferentes técnicas dependiendo de la patología y su estado crónico, siendo las más comunes en los equinos: técnica de aguja seca, electroacupuntura, acuapuntura y acupresión (Scognamillo y Becharall, 2010; Schoen, 2000), también existe la moxibustión, estimulación con láser e implantes (Angeli y Fernandes, 2007; Scognamillo y Becharall, 2010).

Por lo anterior, el objetivo de este artículo es conocer la acupuntura y su aplicabilidad en las alteraciones del sistema musculo-esquelético de los equinos; además de profundizar en las diferentes técnicas empleadas describiendo sus principios fundamentales e importancia en el tratamiento de los trastornos mencionados.

\section{ACUPUNTURA EN MEDICINA VETERINARIA}

\section{Origen}

La AP en medicina veterinaria tiene su origen hace 3000 años, donde se practicaba en animales especialmente en caballos, Shun Yong (480 AC) es la primera persona reportada en hacerlo, siendo considerado el padre de la medicina veterinaria en China. En occidente el uso de acupuntura en animales se inició en Francia a finales de los años 1700 y comenzando el 1800; en el siglo XVIII se practicaba la acupuntura en la medicina veterinaria en Japón y casi todo Asia (Scognamillo y Becharall, 2010). En 1973 la AP veterinaria se reactiva en Norteamérica y en 1974 se crea la Sociedad Internacional de Acupuntura Veterinaria con sus siglas en ingles IVAS (International Veterinary Acupuncture Society) para promover la excelencia en la práctica de la AP veterinaria, sin 
embargo, en 1975 Cuba dio inicio a su verdadero desarrollo; en 1979, la Organización Mundial de la Salud (OMS) aprobó la AP por sus demostraciones y evidentes resultados científicos.

\section{Generalidades}

En base a la Medicina Tradicional China (MTC) los acupuntos se ubican en meridianos o canales, los cuales se conectan con relaciones funcionales específicas, es decir con los órganos y tejidos corporales (Cobos, 2013). La AP se emplea en todo el mundo como única opción o a veces se combinan las terapias con la medicina occidental, para tratar muchas enfermedades en todas las especies domésticas y exóticas, siendo muy empleada en equinos para el tratamiento de cojeras y cólicos (Silva, 2013).

La AP es un método terapéutico que utiliza la estimulación de ciertos puntos cutáneos escogidos, con el fin de producir efectos: analgésicos, antiespasmódicos, descongestionantes e inmunológicos que ayudan a aumentar la capacidad de resistencia del organismo frente a las diversas agresiones (Cobos, 2013). La localización exacta de los acupuntos en los equinos ha sido debatido en las últimas dos décadas, algunos veterinarios se basan en la localización de los acupuntos en los mapas de la acupuntura de la Medicina Veterinaria Tradicional China (MVTC), en la cual no se reconocen los meridianos en animales pero en su lugar han basado sus nombres sobre la localización anatómica o su función (Schoen, 2000); otros veterinarios reciben su entrenamiento a través de la IVAS, enfocándose en el método transposicional, el cual extrapola y traspasa los meridianos humanos al equino, ajustando la localización del punto a la anatomía del equino.

Las principales indicaciones de la AP en la medicina veterinaria se hacen principalmente en enfermedades: musculo-esqueléticas, cutáneas, endocrinas, reproductivas, alteraciones neurológicas, síndrome de malabsorción y del tracto digestivo; pudiéndose aplicar en casi todas las patologías, obteniendo respuestas 
mayores o menores, dependiendo de la enfermedad y de la respuesta del paciente (Silva et al., 2008).

Estudios clínicos y reportes experimentales indican que si se emplea adecuadamente, la AP puede ser segura y efectiva para las terapias de las condiciones musculo-esqueléticas (Haussler, 2007); sin embargo, está contraindicada en animales asustados, con hambre, caquéxicos, agotados y seniles; al igual que en estado de gestación puesto que se puede producir un aborto por inducción de contracciones uterinas (Foganholli et al., 2007; Yamate, 2009), también está contraindicada en áreas tumorales y/o infectadas. También está contraindicada el empleo de la AP antes de confirmar un diagnóstico, porque puede enmascarar los signos y por tanto se puede errar en el diagnóstico definitivo (Scognamillo y Becharall, 2010). Dependiendo de la enfermedad a tratar y si es o no crónica, el número de tratamientos oscila entre 4 a 6 sesiones con intervalos de una semana (Foganholli et al., 2007), con una duración de 5 a 10 minutos por cada sesión (Angeli y Fernandes, 2007); es de aclarar que se necesita entrenamiento en AP para garantizar que el paciente sea tratado con el más mínimo riesgo de contraer reacciones adversas (Yamate, 2009).

\section{Energia Qi}

Se denomina así, aquella energía que es tomada de los elementos como: aire, alimento y las interrelaciones con la naturaleza; el Qi o Chi puede definirse como algo impalpable, pero que está presente en todo, y es la relación existente entre lo material y lo inmaterial; además el Qi circula por los meridianos, regulando la circulación de la sangre, digestión, autoprotección del organismo y todas las actividades vitales (Casasola, 1999). Desde la perspectiva de la MVTC se considera que la salud del animal se verá influenciada por el flujo Qi en su cuerpo, en combinación con las fuerzas universales del Yin y Yang; si el flujo de Qi es insuficiente, desequilibrado o interrumpido, la enfermedad puede aparecer, entonces la AP puede ser utilizada para restablecer ese flujo de energía, aliviando los signos (Pelham et al., 2001). 


\section{Acupuntos}

Son considerados la puerta de entrada y salida de la energía de un organismo (Faria y Scognamillo, 2008), son zonas específica o puntuales del cuerpo: externos o internos, habitualmente ubicados sobre la superficie corporal, siendo sensibles a diferentes estímulos que al puncionar, calentar o presionar el acupunto, curan o alivian los síntomas de la enfermedad (White, 2009); esto se debe a que están situados en depresiones superficiales donde hay uniones neuromusculares y sus áreas cutáneas tienen alta densidad de terminaciones nerviosas libres, además se encuentran: plexos nerviosos, mastocitos, vasos linfáticos, arteriolas y vénulas (Angeli y Fernandes, 2007; Hummel, 2009). Debido a este contexto anatómico se genera una respuesta que afectará inicialmente a la zona estimulada, y por otro lado al meridiano que lo involucra. Los acupuntos son áreas sobre la piel que tienen una resistencia eléctrica disminuida o una conductividad aumentada (Schoen, 2000), y su superficie aproximada es de 10 $\mathrm{mm}^{2}$, localizados por referencias cartográficas establecidas por la tradición china (Foganholli et al., 2007). Los acupuntos están localizados en cuatro estructuras neurales conocidas (Hummel, 2009; Xie y Preast, 2007b):

1. Cerca al punto donde el nervio entra al musculo y cuando se aplica un estímulo eléctrico produce una contracción máxima con una intensidad mínima, teniendo una acción motora.

2. En los nervios periféricos en el plano sagital sobre la línea media dorsal y ventral.

3. En focos de alta densidad nerviosa y en plexos nerviosos; por ejemplo el acupunto GB-34 se ubica donde se divide el nervio peroné común en las ramas superficial y profunda.

4. En la unión del tendón con el musculo.

Cada acupunto tiene una o varias funciones que cuando es estimulado o combinado con otro, como resultado pueden modificar la acción del órgano a tratar; sin embargo, la estimulación errada de los acupuntos, puede no tener 
ningún efecto o provocar el agravamiento de los signos clínicos durante 48 horas aproximadamente (Foganholli et al., 2007).

\section{Meridianos}

En la AP hay varios canales o meridianos de los cuales 12 son pares y se denominan ordinarios (MO), recorren el cuerpo, unen los órganos y vísceras a la superficie del cuerpo; y dos canales extraordinarios impares (ME) que es por donde circula la energía Qi (Taba 1) (Faria y Scognamillo, 2008), cada meridiano tiene un nombre de acuerdo al área del cuerpo o sistema orgánico que representa (Luckenbill, 2005a), a excepción de los ME que coordinan y equilibran el Qi y la sangre de los MO (Xie y Preast, 2007b).

Tabla 1. Meridianos de cuerpo

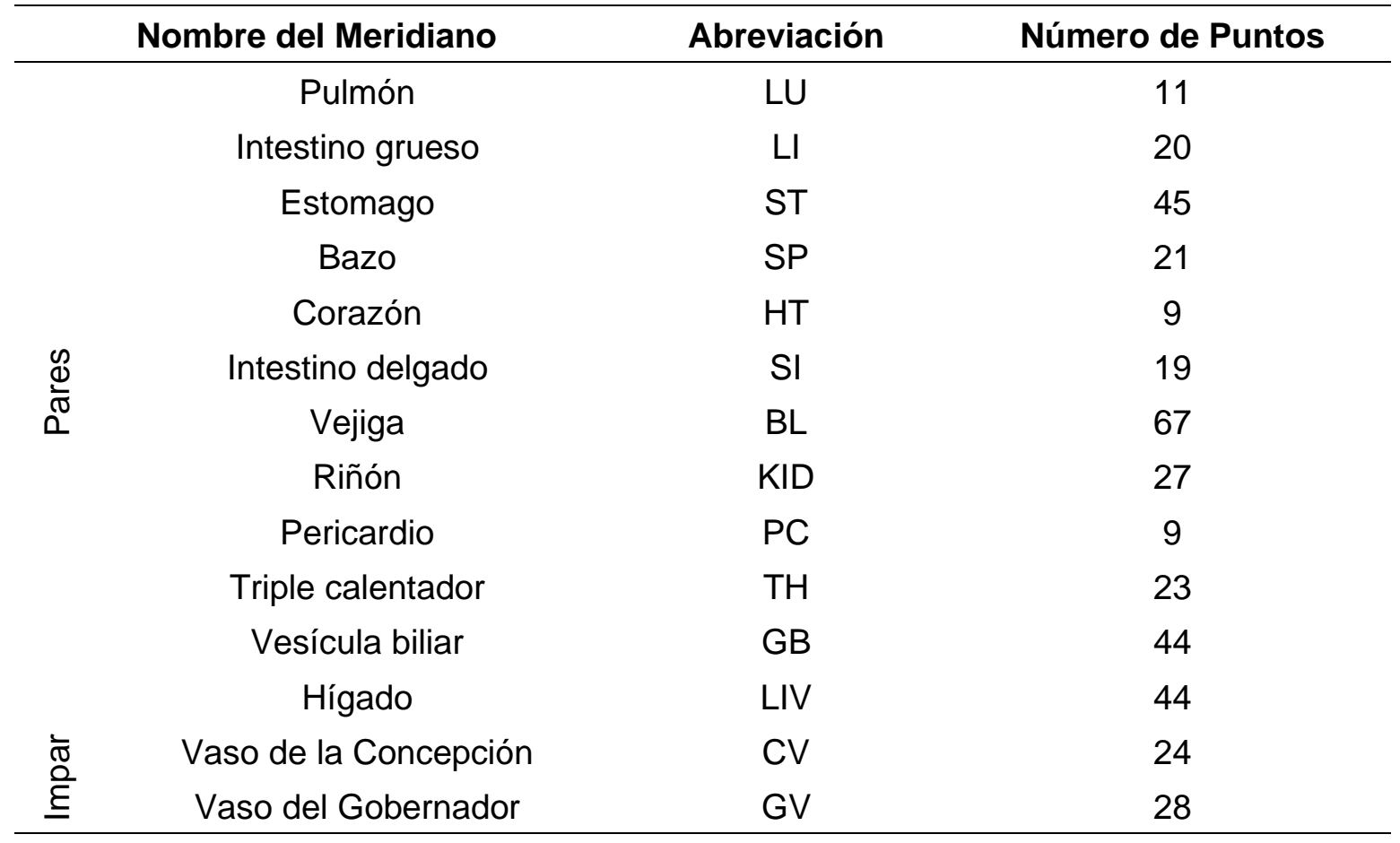

A lo largo de cada meridiano hay acupuntos que han sido identificados por la MVTC, que ante cualquier tipo de estímulo tienen una respuesta, modificando el funcionamiento del órgano o víscera (Luckenbill, 2005a): en el cuerpo la energía Qi fluye a través de estos meridianos que comunican los órganos y las extremidades, y es por medio de las funciones fisiológicas del organismo que es 
regulada y por tanto mantiene un equilibrio (Angeli y Fernandes, 2007), cuando la energía es bloqueada o estancada en algún punto de estos meridianos, se producirá un desequilibrio y la generación de una enfermedad de acuerdo a la localización del bloqueo (Foganholli y Filadelpho, 2007).

\section{TÉCNICAS DE ACUPUNTURA}

Existen diferentes técnicas para estimular los acupuntos que permiten restablecer el equilibrio del Qi en el cuerpo, siendo en equinos las más utilizadas: la de aguja seca, electroacupuntura, acuapuntura y acupresión (Scognamillo y Becharall, 2010; Schoen, 2000); también existe la moxibustión, estimulación a laser e implantes (Tabla 2) (Angeli y Fernandes, 2007); Es importante conocer la fisiología energética y tener en cuenta la anatomía del animal para no dañar vasos importantes, también es conveniente que los animales estén tranquilos durante la sesión, para eso se puede punzar el punto intermedio entre ambos ojos o el acupunto GV-20, en la cima de la cabeza, puesto que los animales quedan totalmente relajados una vez masajeado ese punto (Casasola, 1999).

\section{Agujas en acupuntura}

Las agujas de la AP deben resistir a la corrosión causada por los fluidos corporales, además de la esterilización y los diferentes esfuerzos (tensión, torsión y otros) que son mayores a los que soporta una aguja hipodérmica, porque son más largas y menos afiladas, es por esto que las agujas son de acero inoxidable con mangos de aluminio o cobre (Tabla 3) (Sussmann, 2007).

Las agujas se insertan en las formas que se muestran en la Figura 1, pero en la mayoría de los casos se hace formando un ángulo de $90^{\circ}$ con la superficie del cuerpo, también se en un ángulo de $45^{\circ}$ con preferencia en los puntos donde los músculos son delgados o en zonas próximas a órganos importantes, y la inserción transversal u horizontal, formando un ángulo de $15^{\circ}$ a $25^{\circ}$ está indicada en zonas donde la musculatura es escasa (Casasola, 1999); con la correcta manipulación de las agujas se puede lograr efectos de sedación o tonificación de un punto (Tabla 4). 
Tabla 2. Técnicas de acupuntura más utilizadas en medicina veterinaria

Técnica

Aguja seca

Acupuntura con láser

Acupresión

Moxibustión

Sangrado o hemoacupuntura

Acuapuntura

Implantes

\section{Descripción}

Consiste en la punción de los puntos con agujas, se considera: la naturaleza de la dirección de la punción, la profundidad y la manipulación de la misma; y tiene efectos locales y generalizados (Faria y Scognamillo, 2008; Lutz, 2004).

Electroacupuntura Es un estímulo eléctrico con las agujas, mediante dispositivos se ajusta la frecuencia e intensidad de la corriente, es una alternativa a la punción seca que tiene la ventaja de ahorrar tiempo; se utiliza para aliviar dolores, estimulación nerviosa y desordenes diagnosticados en medicina interna, entre otros (Faria y Scognamillo, 2008; Lutz, 2004; Scognamillo y Becharall, 2010; Yamate, 2009).

Su acción puede ser puntual o con efecto prolongado por la retransmisión nerviosa en el acupunto; se utilizan dos tipos de laser: emisores de luz roja (longitud de onda 632-650 nm, de helioneón) y emisores de luz infrarroja (longitud de onda $902 \mathrm{~nm}$, diodo arsenito de galio) (Lutz, 2004; Yamate, 2009).

Se realiza con los dedos en forma de masaje en acupuntos determinados, teniendo un impacto generalizado en el tratamiento (Faria y Scognamillo, 2008; Scognamillo y Becharall, 2010).

Existen dos tipos: directa e indirecta, en la primera se aplica el estímulo calórico (dispositivo eléctrico) y en la segunda una banda de algodón inmersa en vinagre se envuelve alrededor de la zona y para la generación de calor se emplea el tabaco o picadura de artemisa (moxa), esta técnica es útil para el tratamiento de afecciones crónicas (Scognamillo y Becharall, 2010).

Es una técnica muy empleada en veterinaria, siendo su objetivo causar pérdidas leves de sangre (gotas o pocos milímetros) se realiza punción y sangrado en los acupuntos localizados sobre las venas (tocando únicamente los capilares), empleándose para tratar dolores agudos (Scognamillo y Becharall, 2010).

Consiste en inyectar líquido con una jeringa hipodérmica (Lutz, 2004) con el fin de reforzar puntos alrededor de las agujas, está contraindicada en sitios donde se insertan agujas completas (abdominales ó ventrales) o para animales activos; se utiliza solución salina estéril, vitaminas del complejo $\mathrm{B}$, medicamentos homeopáticos, antibióticos, y en algunos pacientes su propia sangre (Faria y Scognamillo, 2008; Yamate, 2009).

Inyección de una sustancia sólida para proveer una estimulación permanente en acupunto, se utiliza: grano o alambre de oro, granos o bolitas magnéticos (Scognamillo y Becharall, 2010; Yamate, 2009), se utiliza en osteoartritis, epilepsia y enfermedad del disco intervertebral (Faria y Scognamillo, 2008). 


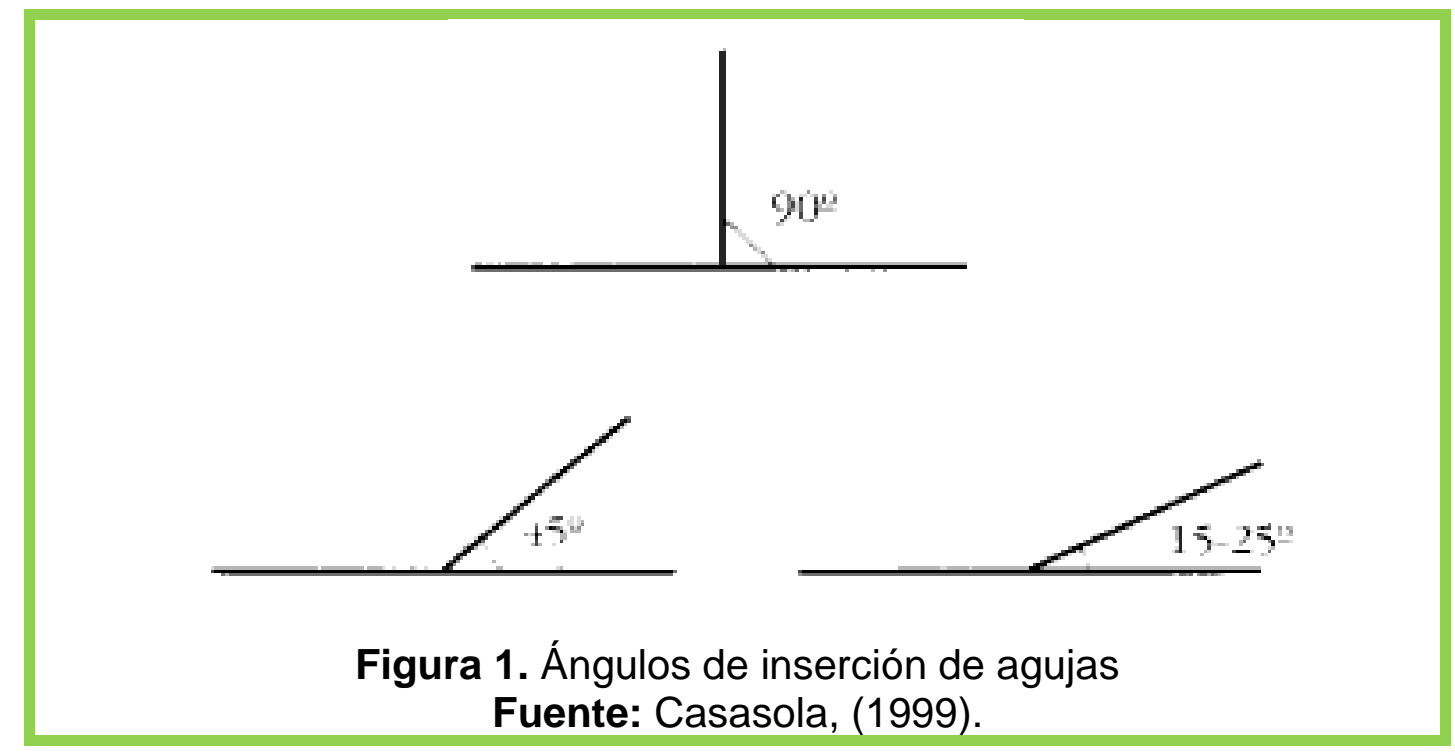

Tabla 3. Agujas empleadas en acupuntura en los equinos

\begin{tabular}{|c|c|c|c|}
\hline Diámetro & Calibre & Longitud & Uso \\
\hline $0.3-0.4 \mathrm{~mm}$ & $28-30 \mathrm{G}$ & $13 \mathrm{~mm}$ (1/2 pulg) & Extremidades \\
\hline $0.3-0.4 \mathrm{~mm}$ & $28-30 \mathrm{G}$ & 25 mm (1 pulg) & $\begin{array}{c}\text { Extremidades, inferiores, } \\
\text { cabeza. }\end{array}$ \\
\hline $0.3-0.4 \mathrm{~mm}$ & $28-30 \mathrm{G}$ & $40 \mathrm{~mm}\left(1 \frac{1}{2}\right.$ pulg $)$ & Tronco, espalda (ponis). \\
\hline $0.3-0.4 \mathrm{~mm}$ & $28-30 \mathrm{G}$ & 50 mm (2 pulg) & Tronco y espalda. \\
\hline $0.3-0.4 \mathrm{~mm}$ & $28-30 \mathrm{G}$ & 75 mm (3 pulg) & Hombros y cadera. \\
\hline
\end{tabular}

Fuente: Yamate. (2009).

\section{Nomenclatura de los acupuntos}

Hay dos sistemas de AP usados en animales: AP en la MTC, una forma de AP basado en los textos de MVTC, el cual es pobremente desarrollado en animales en base a la acupuntura para humanos, dificultando su aprendizaje y uso, porque los acupuntos están aislados uno del otro, cada uno tiene un nombre con un significado anatómico, fisiológico, terapéutico o simbólico; y la AP transposicional veterinaria basada en la teoría y práctica de la AP humana, por medio de códigos que se identifican de acuerdo al canal donde se encuentra localizado, siendo esta última la más usada en la medicina occidental (Faria y Scognamillo, 2008; Xie y Preast, 2007b). 
Tabla 4. Efectos de la manipulación de la aguja

\begin{tabular}{|c|c|}
\hline Tonificación & Sedación \\
\hline Movimientos suaves y gentiles & Movimientos rápidos y bruscos \\
\hline $\begin{array}{l}\text { Rotación en sentido de las manecillas del } \\
\text { reloj }\end{array}$ & $\begin{array}{l}\text { Rotación en sentido contrario a las } \\
\text { manecillas }\end{array}$ \\
\hline Insertar y manipular durante la exhalación & Insertar y manipular durante la inhalación \\
\hline Insertar en la misma dirección del flujo Qi & Insertar en dirección contraria al flujo $Q i$ \\
\hline Utilizar agujas de oro & Utilizar agujas de plata \\
\hline Sangrar un poco & Sangrar en gran cantidad \\
\hline Corta duración (5-10 minutos) & Larga duración (15-20 minutos) \\
\hline
\end{tabular}

Fuente: Builes, (2000).

\section{MECANISMOS DE ACCIÓN DE LA ACUPUNTURA}

La acupuntura puede ser definida como la estimulación de puntos predeterminados sobre el cuerpo para lograr un efecto terapéutico o hemostático (Angeli y Fernandes, 2007; Schoen, 2000). La MVTC ha explicado esos efectos por cuatro mil años basados en observaciones empíricas y descripciones de fenómenos naturales, mientras que la medicina occidental incluye teorías neural no opioide, autonómica, mecanismos humorales o teorías bioeléctricas (Tabla 5) (Chan et al., 2001).

Un estudio realizado en equinos con cojeras donde empleó la electro-acupuntura (EA), reveló que las concentraciones plasmáticas de $\beta$-endorfina aumentaron después de los estímulos, y simultáneamente disminuyó la cojera del paciente evaluado (Xie et al., 2001). Las $\beta$-endorfinas, encefalinas y dinorfinas son liberadas por medio de la EA en dependencia de la frecuencia de la estimulación, a bajas frecuencias $(2-4 \mathrm{~Hz})$ se liberan las $\beta$-endorfinas y a altas $(100 \mathrm{~Hz})$ las dinorfinas que interactúan a nivel de la corteza cerebral y la médula espinal (Cabana y Ruiz, 2004). Se ha relatado que la AP y el ejercicio poseen efectos fisiológicos muy semejantes en el organismo: ambos estimulan las fibras nerviosas aferentes, producen efectos similares en los sistemas cardiovasculares y 
pulmonares, y producen respuestas neuroendocrinas semejantes (Pelham et al., 2001).

Tabla 5. Teorías sobre el mecanismo de acción de la acupuntura

\begin{tabular}{|c|c|}
\hline Teoría & Mecanismo de Acción \\
\hline $\begin{array}{l}\text { Neural no } \\
\text { opioide }\end{array}$ & $\begin{array}{l}\text { Implica la inhibición de impulsos conducidos por ciertas fibras nerviosas, } \\
\text { por lo tanto el efecto de la AP dependería del sistema nervioso central. }\end{array}$ \\
\hline Humoral & $\begin{array}{l}\text { Se basa en la evidencia de que la AP estimula la liberación de opioides } \\
\text { endógenos, este mecanismo actúa en varias partes del SNC, inhibiendo } \\
\text { la percepción dolorosa y la transmisión del dolor de la médula espinal } \\
\text { por medio de la inhibición descendente. }\end{array}$ \\
\hline Bioeléctrica & $\begin{array}{l}\text { Demuestra que existe una conducción diferente a la nerviosa, incluyendo } \\
\text { partes del SNC; en la AP los canales de energía poseen menor } \\
\text { resistencia que la piel adyacente, permitiendo el flujo de una corriente } \\
\text { bioeléctrica por medio de ellas; éste fenómeno de propagación de } \\
\text { energía ha sido muy bien documentada por modelos neurofisiológicos. }\end{array}$ \\
\hline Autonómica & $\begin{array}{l}\text { La estimulación cutánea por agujas es transmitida para las vísceras por } \\
\text { sinapsis somatovisceral en la médula espinal; cuando determinado } \\
\text { órgano sufre alteraciones fisiopatológicas, uno o más acupuntos } \\
\text { relacionados pueden estar sensibles o mostrar signos de anormalidades. }\end{array}$ \\
\hline Neural & $\begin{array}{l}\text { Los neurotransmisores intervienen en la transmisión del estímulo } \\
\text { doloroso como la sustancia P, serotonina (dolor crónico), acido- } \\
\text { gammaaminobutírico (GABA) y la noradrenalina (dolor agudo); siendo } \\
\text { modificada su liberación mediante la AP interfiriendo en la conducción de } \\
\text { dicho estimulo (Cabana y Ruiz, 2004). Hay estudios que demuestran que } \\
\text { la AP actúa estimulando repetidamente las terminaciones nerviosas } \\
\text { superficiales y profundas del cuerpo del animal, de esta manera se } \\
\text { alivian dolores locales y distantes, que pueden ser crónicos o agudos; } \\
\text { con estos estudios se desmiente que la AP funcionaba solo por el efecto } \\
\text { placebo generado por la autosugestión e hipnosis (Foganholli et al., } \\
\text { 2007). }\end{array}$ \\
\hline
\end{tabular}

El efecto deseado con el estímulo de un acupunto se caracteriza, por la inducción de la inflamación aséptica, estímulo directo de: los nervios de la piel, del tejido perivascular, los tendones y músculos, activación del mecanismo inhibitorio del dolor por la liberación de endorfinas y hormonas adenocorticotróficas (ACTH), lo cual mejora la circulación local, liberación de serotonina, inducción de efectos humorales e inmunomodulacion trombocitaría (Angeli y Fernandes, 2007), esto porque el estímulo es transmitido a los centros nerviosos (Foganholli et al., 2007), induciendo una serie de cambios en la vía ascendente del dolor y del sistema de 
inhibición descendente afectando la activación interneural en la medula espinal, y así previniendo la conducción de mecanismos que intensifican el dolor en la corteza (Cabana y Ruiz, 2004). Estos impulsos serán codificados, analizados, memorizados e integrados en otros circuitos, y conforme a su intensidad, localización y naturaleza, la respuesta será benéfica (Foganholli et al., 2007); también la AP activa el núcleo magno del rafe y estimula el hipotálamo, atenuando la percepción dolorosa (Cabana y Ruiz, 2004).

Estudios histológicos han revelado que los acupuntos consisten en un adelgazamiento de la piel debido a una modificación de las fibras de colágeno, presentando terminaciones nerviosas, arteriolas y vénulas que penetran a través de la fascia de los acupuntos (Hummel, 2009; Schoen, 2000).

\section{ALTERACIONES DEL SISTEMA MUSCULO-ESQUELÉTICO Y NERVIOSO}

En etiologías de dolor crónico del sistema musculoesquelético, la AP es útil para estimular el sistema inmune, puesto que induce una respuesta de este sistema, incluyendo la inmunidad humoral, incremento de las células sanguíneas blancas, de la actividad fagocítica y los niveles de anticuerpos, y aumenta los niveles de interferón, todo esto debido al aumento de la microcirculación (Ridgway, 2005).

\section{Cojeras}

La AP es una excelente ayuda para el diagnóstico adjunto al examen convencional de las claudicaciones, el diagnóstico está basado en el nivel de sensibilidad a la palpación de determinados acupuntos (Schoen, 2000; Shmalberg y Xie, 2009), muchos de estos se localizan lateral a la línea media dorsal entre los músculos iliocostales y larguísimos, conocido en la acupuntura transposicional como el meridiano de la vejiga; cada acupunto puede tener de cuatro a cinco diagnósticos de acuerdo a la reacción que presenta el paciente durante el examen (Schoen, 2000), por ejemplo, el punto LI-16 puede ser reactivo en una cojera del miembro anterior, la sensibilidad del acupunto en el meridiano de la vejiga a lo largo del dorso, puede indicar que es una cojera del miembro posterior relacionado con el corvejón o la rodilla, o con problemas de silla o de conformación, mientras que una 
reactividad exagerada podría indicar problemas de órganos internos vía reflejo somatovisceral. La AP ha sido exitosa en terapias de numerosas cojeras equinas incluyendo las de problemas dorsales crónicos, problemas del corvejón o rodilla, laminitis, enfermedad del navicular, y también varias injurias en el tejido blando (Schoen, 2000).

El objetivo del tratamiento con AP es reducir el dolor, generando un efecto relajante de los músculos locales, liberando endorfinas y/o estimulando el suministro sanguíneo (Luckenbill, 2005a). Xie et al., (2001) en un modelo experimental encontraron que la electroacupuntura disminuyó las cojeras, reduciéndolas significativamente, por el incremento de las concentraciones plasmáticas de $\beta$-endorfina, indicando que su liberación puede ser una de las vías en la cual la AP alivia el dolor.

Las cojeras pueden generarse por alteraciones de diferentes estructuras anatómicas (ligamentos, tendones, hueso y/o músculos) localizadas a lo largo del miembro; algunos acupuntos muestran los efectos clínicos de estas condiciones patológicas, para cubrir estas áreas se pueden seleccionar, para: esqueleto BL-23 y BL-11, tendones GB-34 y BL-18, y músculos BL-20; adicionalmente para el tratamiento de cojeras de los miembros anteriores se emplean los acupuntos SI-9, SI-3 y TH-1; y para los miembros posteriores BL-54, BL-67 y ST-45 (Shmalberg y Xie, 2009). Autores como Luckenbill, (2005a) emplean la siguiente fórmula para tratar las cojeras sin importar su origen (Figura 2):

- Cojeras de los miembros posteriores asociados al dolor del dorso: BL-23, BL25, BL-27, BL-30, GB-30, GB-33, BL-37, y el punto extra arriba de la tuberosidad coxal; si el dolor está en la articulación de la rodilla se adiciona LIV-8 y KID-10.

- Dolor del tren posterior: Tratar los puntos usados en cojera del miembro posterior, más BL-13 y BL-18.

- Cojera de miembro anterior y dolor de hombro: Usar los acupuntos LU-1, BL13, LI-16, TH-1 y SI-9. 
- Dolor artrítico general en caballos viejos: Usar la combinación apropiada para cojeras de miembros anteriores y posteriores y adicionar BL-11, BL-40 y PC-6.

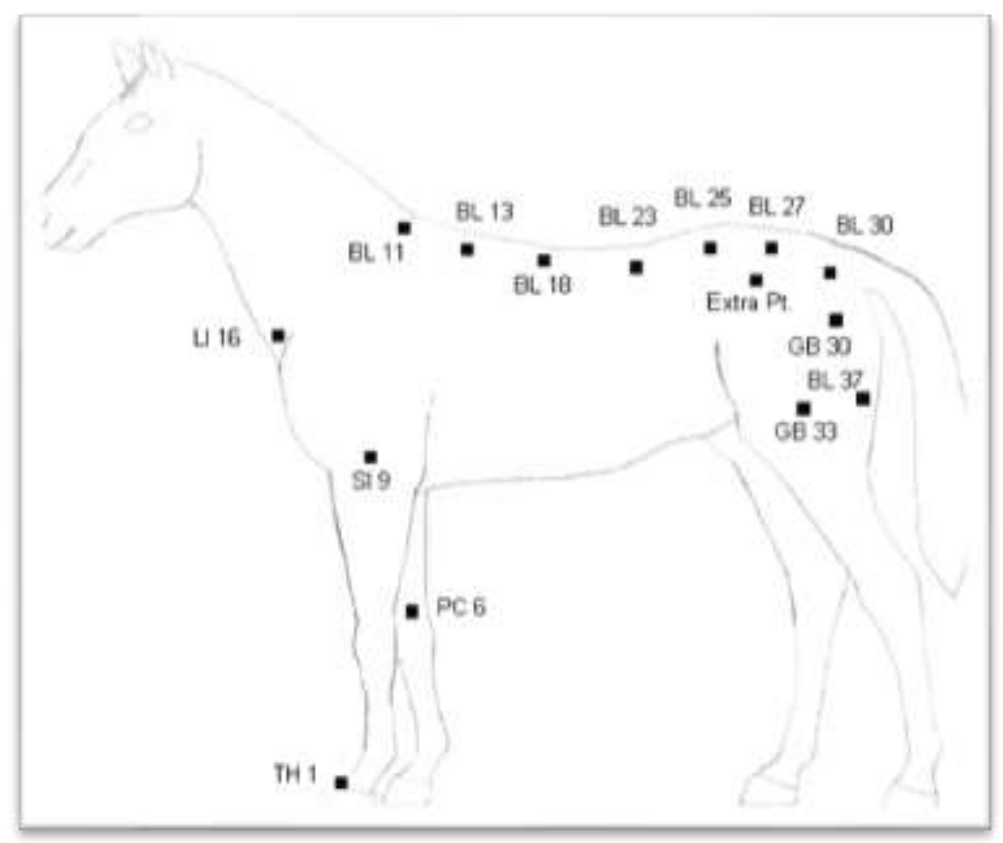

Figura 2. Acupuntos empleados usualmente para el tratamiento de cojeras.

Fuente: Luckenbill, (2005a).

Mientras Xie y Preast, (2007a) tratan las cojeras de acuerdo a su origen, como en las originadas en el hombro por el estancamiento de la sangre y del Qi en esta región y en el pecho, esto se denomina en la MTVC como Xiong-bo-tong por bloqueo del Qi y la sangre en el pecho, que puede ocurrir por deslizamiento, salto, ejercicio agotador o falta de este, en el cual se pueden emplear diferentes acupuntos con diferente técnica en el tratamiento (Tabla 6); mientras que para esta alteración Luckenbill, (2005a) emplea los acupuntos LU-1, BL-13, LI-16, TH-1 y SI-9, insertando las agujas a $4-5 \mathrm{~cm}$ de profundidad, con repetición semanal o una mensual dependiendo de la severidad.

Los Acupuntos empleados por Xie y Preast, (2007a) en el tratamiento de cojeras son:

- Hombro: Técnica de aguja seca en los acupuntos LI-1, TH-1 y SI-1; hemoacupuntura en SI-3 ó Xiong-tang; acupuntura en SI-9, SI-10, TH-14, LU1, Yan-zhou y Cheng-deng; electroacupuntura si hay parálisis del nervio radial 
en GB-21, SI-9, LI-14, LI-15, LI-10 y LI-11; neumoacupuntura si hay atrofia muscular en Gong-zi.

- Codo: TH-10, SI-8, Zhou-shu, Yan-zhou, Cheng-deng, SI-3, LI-4, LI-1, TH-1 y TH-3; y los puntos del síndrome Bi óseo.

- Articulación carpiana: Aguja seca en LI-1, TH-1 y LU-11; acupuntura o electroacupuntura en TH-4, TH-5, PC-6, BL-11, BL-23 y SI-9; y hemoacupuntura en condiciones agudas en acupunto SI-3.

- Tercer carpiano: Aguja seca en TH-1, SI-1, SI-3, SI-4, LI-4, HT-8; y electroacupuntura o acuapuntura en SI-9, GB-21, BL-11 y BL-23.

- Segundo y cuarto metacarpiano: Aguja seca en TH-1, SI-1, SI-3, LU-11, LU10, LI-4, LI-11, LU-1 y PC-8; y electroacupuntura o acuapuntura en LI-15, GB21, BL-11, BL-23 y Shen-shu en combinación con un plan de entrenamiento.

- Espolón y cuartilla: En miembro anterior: LI-1, TH-1, SI-1, Ming-tang, SI-3, LI-4 y SI-9; y miembro posterior: ST-45, GB-44, SP-3, BL-67, BL-66, BL-67, GB-42, GB-43 y Lao-Tang.

- Talón y casco: Aguja seca o acuapuntura en BL-18, BL-19, BL-11 y Bai-Hui; aguja seca en miembro anterior: TH-1 y HT-9, y en posterior: LIV-1 y LIV-3; electroacupuntura $20 \mathrm{~Hz}$ durante 20 minutos en miembro anterior: LI-1+LI-3, SI-1+SI-3, LU-11+LI-15, GB-21+TH-15 y SI-9+TH-14, y en posterior: Hou-timen, KID-1, BL-67+BL-65, GB-44+GB-42, SP-1+SP-3, BL-54 y Shen-shu.

- Cadera: Aguja seca o acuapuntura en Bai-hui y Yan-chi; aguja seca en GB-44, ST-45 y BL-67/60; acuapuntura o electroacupuntura en Shen-shu, Shen-jiao, BL-54 y Lu-gu; y neumoacupuntura cuando hay atrofia muscular en BL-54 y BL-36.

- Corvejón: aguja seca en Bai-hui, BL-67, GB-44, LIV-1 y KID-1; y electroacupuntura o acuapuntura en Yan-chi, Shen-jiao, Dan-tian, Ju-liao, BL54, BL-35, BL-39 y BL-60.

- Rodilla: Aguja seca en Bai-hui, ST-45 y GB-44; acuapuntura BL-36, BL-37 y BL-38; y electroacupuntura en BL-54, BL-20 ó BL-21 bilaterales, Dan-tian + Julio, ST-34 + GB-34, SP-10 + ST-34 y Yang-ling + BL-40; en injurias de ligamentos adicionar: LIV-8, GB-32, GB-33, LIV-3 y LIV-8; en bloqueo rotuliano 
adicionar: ST-35, ST-32, Hou-fu-tu, Xiao-kua; y quistes óseos adicionar: BL11, BL-23, KID-3 y KID-10.

Otro causante común en la presentación de cojeras son los desórdenes del Jin, lo cual se refiere a tendones, ligamentos, fascia y miofascia; las alteraciones incluyen injurias en los ligamentos, daño en el ligamento suspensorio, ruptura de los ligamentos cruzados, tendinitis, tendones contraídos y tendosinovitis; el Jin hace referencia a los principales tejidos entrelazados de las articulaciones y músculos, siendo fundamental para el movimiento de los miembros; una deficiencia en sangre hepática o Jin predispone a injurias en tendones y ligamentos, debido a entrenamientos inapropiados, ejercicio excesivo, herraje inapropiado y desgaste excesivo de la parte posterior del casco; estas injurias bloquean el flujo Qi, que se estanca ocasionando dolor y por consiguiente las cojeras (Xie y Preast, 2007a). La AP puede dar un alivio rápido a la inflamación, calor y dolor en una tendinitis, pero muchos expertos agregan que a largo plazo los resultados pueden ser pobres, porque en estos casos lo ideal es mantener el paciente en reposo hasta su recuperación completa (Luo, 2016).

El tratamiento para la cojera relacionado con problemas en los tendones y ligamentos consta de los acupuntos GB-34, BL-18/19, BL-17, SP-10, BL-23, KID3, LIV-3 (Xie y Preast, 2007a); además se puede adicionar estímulos en los siguientes casos:

- Tendón encorvado o contraído del miembro anterior: AP en LI-4, TH-1, TH-2, TH-3, SI-3, PC-9, PC-8, LU-10, HT-7, Xi-mai, Ming-tang, Qian-ti-men.

- Tendón encorvado o contraído del miembro posterior: AP en SP-3, LIV-1, LIV3, ST-45, ST-44, ST-43, KID-1, BL-65, BL-66, ST-67, GB-44, GB-42, GB-43.

- Ligamento cruzado roto: AP en GB-32, GB-33, ST-34, ST-35, ST-36, SP-9, BL-40, LIV-8, KID-1.

- Puntos diagnósticos para problemas de tendón y ligamentos: TH-15, SI-16, BL-14/15 BL18/19. 
Para el diagnostico de una cojera, existen acupuntos como en la cadera: BL-54 ó Lu-gu, BL-53, Huan- tiao, Huan-hou, o Huan-zhong (o GB-29/30), Shen-shu, Shen-peng, Shen-jiao, BL-23 a BL-26, GB-20 o GB-21, Ba-jiao o BL-36 (Tablas 6 y 7) (Xie y Preast, 2007a).

Tabla 6. Acupuntos diagnósticos en los equinos

\begin{tabular}{cc}
\hline Acupunto & Indicaciones \\
\hline LI-18 & Patas (cajón, casco, navicular) \\
PC-1 & Patas (cajón, navicular, cascos) \\
LI-16 & Espolón \\
TH-15 & Ligamento suspensorio frontal \\
Hua-tuo-jia-ji en C4 & Carpos \\
LI-15 & Hombro \\
BL-54 & Cadera \\
Lu-gu & Cadera \\
SP-11 & Rodilla \\
ST-31 & Rodilla \\
GB.27 & Corvejón \\
Puntos de la vejiga & Toracolumbar \\
\hline
\end{tabular}

Fuente: Shmalberg y Xie, (2009).

La rodilla es una región que contiene uniones bastante complejas con numerosos ligamentos; el dolor en la rodilla se refiere a injurias del tejido blando (rotula y ligamentos colaterales), quistes óseos, elevación de la rótula, enfermedad degenerativa de la articulación de la rodilla y osteocondrosis desecante (Xie y Preast, 2007a); el bloqueo del Qi por dolor en el corvejón, compensación por subluxación sacroilíaca, herraje, ejercicio o entrenamiento inapropiados, estrés generado por el entrenamiento o el medio ambiente, comprometen el mecanismo de defensa del animal. El ataque de viento-frio entra por los meridianos SI y BL, evidenciándose como dolor en el cuello y lumbalgia, a medida que la condición se desarrolla los meridianos Shao yang se ven afectados incluyendo (GB y $T H$ ), experimentando el caballo rigidez en los miembros anteriores y dolor en la cadera, si la invasión continua se vería afectado los meridianos Yang-ming (LI y ST), posteriormente involucrando a los meridianos Tai-yin (LU y SP); en este punto se 
evidencia dolor muscular en los 4 miembros, predisponiendo al paciente a invasión de patógenos; si se produce una infección los meridianos que estarían afectados son el Shao-yin y Yue-yin, pudiendo llevar a infecciones graves como la mielitis protozoaria equina (Xie y Preast, 2007a).

Luckenbill, (2005a) emplea la fórmula para "cojera de los miembros posteriores" con los acupuntos BL-23, BL-25, BL-27, BL-30, GB-30, GB-33, BL-37 y el" punto extra" de la tuberosidad coxal, adicionando dos puntos en el aspecto medial de la unión de la rodilla, SP-9 y KID-10; el autor ha observado una respuesta efectiva en los acupuntos GB-30 y SP- 9, que son benéficos en una inserción profunda.

Tabla 7. Puntos adicionales para tratamiento de cojeras por área afectada

\begin{tabular}{cc}
\hline Acupunto & Indicación \\
\hline PC-9 & Miembro anterior distal \\
PC-6 & Carpos \\
SI-9 & Hombro \\
BL-53 & Cadera \\
ST-36 & Rodilla \\
BL-60 & Tarsos \\
KD-1 & Miembro posterior distal \\
\hline
\end{tabular}

Fuente: Shmalber et al., (2009)

Los acupuntos clave para aliviar el dolor en una luxación rotuliana incluyen: ST-35, $X i$-yan (en el tendón de la rótula entre la tuberosidad de la tibia y el borde inferior de la rótula), ST-34, ST-36, LIV-7, y LIV-8; adicionalmente cuando se sospecha que hay debilidad del musculo vasto medio y éste afecta la rótula, emplear los acupuntos SP-10, KI-3, KI-10 y SP-6 (Wynn y Marsden, 2003).

\section{Rabdomiólisis}

Azoturia o miositis común en potros de carrera, en el que está principalmente involucrado un desbalance de los elementos tierra-agua-madera, lo cual incide porque los cambios climáticos súbitos (días de frio, humedad, y vientos) o tiempo de primavera (madera), un desbalance en la dieta (muchos carbohidratos) y 
ejercicio (irregular o poco) conducen a estancamiento Qi de los músculos, además el desorden esplénico (estancamiento Qi muscular) puede controlar (cheng) el sistema agua (riñón y vejiga) conduciendo a azoturia; se puede observar dolor muscular y calambres tan pronto inicia le ejercicio, músculos inflamados, firmes y adoloridos, y animales reacios a continuar con la actividad con sensibilidad a la palpación de los acupuntos BL-18 a BL-28, BL-54, Lu-gu, SI-9, SI-10. La AP puede ayudar en la azoturia, pero para obtener mejores resultados se requiere que las anormalidades subyacentes de la función de los órganos (especialmente del hígado) también sean tratadas, sumado a cambios apropiados en la alimentación y practica de entrenamiento de acuerdo a la actividad que ejerce el paciente (Xie y Preast, 2007a).

El tratamiento sugiere mover Qi para aliviar el dolor, mover la sangre para resolver el estancamiento, armonizar el LIV, SP y KID, dos a cuatro tratamientos, cada una a dos semanas, siendo efectivo en $95 \%$; se emplea la técnica de aguja seca en Bai-hui, Wei-ben, Wei-jian, KID-1, LIV-1, ST-1, BL-67; electroacupuntura en BL-18, $\mathrm{BL}-21, \mathrm{BL}-54$ y Shen-shu, en los miembros traseros y delanteros afectados adicionar Lu-gu, GB-30, BL-36, BL-37 y SI- 9, GB-21 y TH-15 respectivamente (Xie y Preast, 2007a).

\section{Dolor del dorso}

Su origen podría ser primario o secundario (como parte de un desorden generalizado), el dolor primario puede ser debido a un malestar del musculo paravertebral, lesiones vertebrales o sacro-ilíacas, o causadas por la silla, también artríticas o procesos de la espina dorsal; el dolor secundario lo haría por alteraciones de los órganos abdominales o torácicos, espasmos de los músculos del dorso del animal en compensación por problemas articulares en los miembros ó como un desorden generalizado (distrofia muscular nutricional por deficiencia de

vitamina $\mathrm{E}$ y selenio, o miositis en azoturia) (Chan et al., 2001). El dolor del dorso agudo posee un carácter nociceptivo en la presentación crónica caracterizado por ausencia de lesiones, porque el dolor es originado por irritación constante de los nervios preganglionares aferentes, como en las fibromialgias en humanos (Alves 
et al., 2007). En la MVTC el hígado, bazo y riñón son importantes en el mantenimiento integral de los tendones, músculos, articulaciones y huesos (Chan et al., 2001). La AP puede ser una terapia única efectiva para lumbalgias crónicas en humanos y animales, y es coadyuvante de otros tratamientos (Alves et al., 2007).

Los caballos se tratan por dos razones: 1) cojeras o muestran signos de dolor o rigidez; y 2) su desempeño disminuye gradualmente o de manera súbita. En ambos casos la terapia convencional usada (masajes, natación y medicación con analgésicos o AINEs) tiene resultados pobres. La palpación de los puntos de diagnóstico BL-17 a BL-30 del canal de la vejiga invocan una respuesta violenta en el paciente, el tratamiento con AP dependiendo de la naturaleza, causa y duración, tiene un éxito entre 70-90\% (Chan et al., 2001). La presión sobre ciertos puntos a lo largo del dorso del caballo, sirve para evidenciar la sensibilidad de las áreas afectadas, se realiza a lo largo del surco creado por los músculos larguísimo lumbar y el lumbar iliocostal, este surco es fácilmente identificado en el caballo y referido en el meridiano de la vejiga (cada acupunto de este meridiano está alrededor de 5-10 cm de la línea media dorsal) (Luckenbill, 2005b).

Luckenbill, (2005b) realiza la AP como una terapia independiente, el cual provee un beneficio de dos a cuatro semanas, sin embargo si el dolor se origina en las articulaciones de la parte distal de los miembros, el beneficio dado por la AP es de tan solo unos pocos días; cuando se emplea el tratamiento para el dolor de espalda con AP, es mejor tratar los puntos que están distales a las áreas afectadas, los canales seleccionados para el tratamiento son BL y GV, que son puntos para el dolor paravertebral (Chan et al., 2001; Shmalberg y Xie, 2009), si la respuesta es difusa y está comprometida la articulación de la cadera, se podría considerar usar los acupuntos GB-30, GB-34 y Bai-hui. De manera general, la técnica de aguja seca y electroacupuntura son usados en puntos para el aliviar el dolor, mientras que la moxibustión se emplea en los acupuntos tradicionales pero en ausencia del dolor (Chan et al., 2001). 
El dolor del dorso inmunomediado se puede atribuir a la inflamación de los nervios periféricos de la región de la cruz, dorso y cadera; los acupunturistas equinos han seleccionado acupuntos para valorar la sensibilidad de éstos durante la palpación, cuando se presenta esta condición, específicamente los acupuntos LU-1, BL-13, SP-9 y el Punto Extra sobre la cadera izquierda; estos acupuntos pueden ser tratados con medicamentos homeopáticos (Luckenbill, 2005b).

Las técnicas que se pueden emplear en AP veterinaria para el tratamiento del dolor del dorso son aguja seca, electroacupuntura, moxibustión, acupuntura con láser (Alves et al., 2007; Chan et al., 2001); estando los acupuntos implicados ubicados a lo largo del meridiano de la vejiga, lo cuales son: BL-11, BL-13, BL-18, BL-23, BL-25 y BL-30 (Luckenbill, 2005b).

La experiencia clínica de Shmalberg y Xie, (2009) ha demostrado que en promedio tres sesiones de AP son necesarios para resolver la cojera o disminuir la injuria muscular; condiciones degenerativas y ortopédicas toman más tiempo y por tanto se debe tener claro que los cambios en la estructura del tendón requieren un mayor número de tratamientos para lograr la recuperación completa.

Builes, (2000) maneja todos los acupuntos localizados en el dorso del animal para el tratamiento de alteraciones localizadas en esta región (BL-14 al BL-34 y el BL41 al BL-54, GV-3 al GV-14 y Bai-hul); teniendo un éxito del 90\% aproximadamente, empleando las técnicas de aguja seca, electroacupuntura, moxibustión, acupuntura con láser, hemoacupuntura, y acuapuntura en casos crónicos, además emplea como terapia adicional la magnetoterapia.

\section{CONCLUSIONES}

La acupuntura es una técnica milenaria que forma parte de la cultura china, por lo cual en occidente puede llegar a ser enigmático e incomprensible, es por esto que para su entendimiento y aprendizaje es necesario conocer parte de su cultura, logrando con ello manejar la acupuntura dentro de los tratamientos empleados de la medicina veterinaria occidental moderna. 
Muchas técnicas existen para emplearse en la acupuntura, destacándose la de aguja seca, electroacupuntura, acuapuntura, acupuntura con láser, acupresión, implantación y moxibustión, que dependiendo de la alteración y de su nivel de afectación en el paciente, se emplea una o varias de estas técnicas en el tratamiento, siendo las más empleadas aguja seca y electroacupuntura, la primera por ser económica y efectiva; y la otra por ser efectiva y tener un efecto más potente por sus estímulos eléctricos, que generan una serie de efectos fisiológicos que son benéficos en la recuperación del paciente.

En las alteraciones del sistema musculoesquelético la acupuntura transposicional es la más usada dentro de los tratamientos instaurados, por su fácil aprendizaje y localización, que se basa en canales de flujo de energía o meridianos, que a lo largo de su recorrido se localizan acupuntos denominados con abreviaturas del mismo nombre, acompañado con un número que va en orden ascendente desde el inicio hasta el final del canal; estos acupuntos se pueden emplear como tratamiento único o en combinación con otros planes, como la medicina homeopática o la medicina alopática. Los resultados son variados de acuerdo a la patología y a su severidad, y es muy útil para el manejo del dolor provocado por alteraciones del sistema musculo-esquelético, siendo ayudante en su recuperación; en su mayoría el éxito se debe a que se instaura como coadyuvante en otras terapias, además mejora la calidad de vida de los pacientes y el desempeño de los animales en especial en aquellos que realizan esfuerzo físico.

\section{REFERENCIAS BIBLIOGRÁFICAS}

1. Alves A.L.G., Fonseca B.P.A.d., Thomassian A., Nicoletti J.L.d.M., Hussni C.A., Silveira A.B.d. Lombalgia em eqüinos. Brazilian Journal of Veterinary Research and Animal Science. 44 (3): 191-199. 2007.

2. Angeli A.L., Fernandes J.G. Acupuntura aplicada à medicina esportiva eqüina. Rev. Acad., Curitiba. 5 (3): 325-333. 2007.

3. Builes J.L., Acupuntura en equinos, Medicina Veterinaria. Facultad de Ciencias Agropecuarias, Universidad de la Salle, Bogotá. Colombia. 2000.

4. Cabana S.J.A., Ruiz R.R. Analgesia por acupuntura. Revista Cubana de Medicina Militar. 33 (1): 2004.

5. Casasola M. Acupuntura en animales: La historia lejana. Mandala Ediciones, Madrid, España. 1999. 
6. Cobos R., R. Acupuntura, electroacupuntura, moxibustión y técnicas relacionadas en el tratamiento del dolor. Revista de la Sociedad Española del Dolor. 20 (5): 263-277. 2013.

7. Chan W.W., Chen K.Y., Liu H., Wu L.S., Lin J.H. Acupuncture for general veterinary practice. Journal of Veterinary Medical Science. 63 (10): 1057-1062. 2001.

8. Faria A., Scognamillo S.M. Acupuntura veterinária: conceitos e técnicasrevisão. Ars Veterinaria. 24 (2): 83-91. 2008.

9. Foganholli J.N., Filadelpho A.L. Tratamento de distúrbios neuromusculares em cães com o uso da acupuntura. Revista Científica Eletrônica de Medicina Veterinária. 5 (9): 1-7. 2007.

10. Foganholli J.N., Rodrigues R.V., Procópio V.A., Filadelpho A.L. A utilização da acupuntura no tratamento de patologias na medicina veterinária. Revista Científica Eletrônica de Medicina Veterinária. 5 (9): 1-7. 2007.

11. Haussler K. Review of the examination and treatment of back and pelvic disorders. En: Focus Meeting of the American Association of Equine Practioners-Lameneses and Imaging. p 158-181. 2007.

12. Hummel J. Os benefícios da acupuntura na medicina veterinária, Médico Veterinario. Facultad de Veterinaria, Universidad Federal do Rio Grande do Sul, Porto Alegre, Brasil. 27 p. 2009.

13. Luckenbill B. The practical use of acupuncture for equine lameness. 2005a.

14. Luckenbill B. Treatment options for navicular disease in the equine patient. 2005b.

15. Luo L. Acupuntura explicada punto por punto. Selector S.A. de C.V., México, D.F. 2016.

16. Lutz E. Points moteurs, points de tension, trigger points, points d'acupuncture: relations, intérêts en diagnostic et thérapeutique chez le cheval, Thèse de doctorat vétérinaire. Université Claude Bernard, Lyon, 2004.

17. Pelham T.W., Hot L.E., Stalker R. Acupuncture in human performance. Journal of Strength and conditioning research. 15 (2): 266-271. 2001.

18. Ridgway K. Diagnosis and treatment of equine musculo-skeletal pain. The role of the complementary modalities: Acupuncture and chiropractic. Proceedings of the... annual convention. 2005. Recuperado 23 Febrero 2016. Disponible En: http://www.ivis.org/proceedings/aaep/2005/ridgway/chapter.asp?LA=1

19. Scognamillo S.M.V.R., Becharall G.H. Acupuntura: histórico, bases teóricas e sua aplicação em Medicina Veterinária. Ciência Rural. 40 (2): 1-10. 2010.

20. Schoen A.M. Equine acupuncture: incorporation into lameness diagnosis and treatment. En: AAEP Proc. 46: 80-83. 2000.

21. Shmalberg J., Xie $\mathrm{H}$. The clinical application of equine acupuncture. Journal of equine veterinary science. 29 (8): 645-652. 2009.

22. Silva B.F. Aplicación de la acupuntura para el diagnóstico y tratamiento de claudicaciones en equinos, Doctor en Ciencias Veterinarias. Facultad de Veterinaria, Universidad de la República, Montevideo, Uruguay. 40 p. 2013.

23. Silva D.T., Alves G.C., Filadelpho A.L. Medicina alternativa: acupuntura e quiropraxia aplicadas em Medicina Veterinária. Revista Científica Eletônica de Medicina Veterinária. 6 (11): 1-5. 2008. 
24. Sussmann D.J. Breve historia de la acupuntura. Editorial Kier, Buenos Aires, Argentina. p 22-32. 2004.

25. Sussmann D.J. Acupuntura: Teoría y práctica: La antigua terapéutica china al alcance del medio practico. Editorial Kier, Buenos Aires, Argentina. 416 p. 2007.

26. White A. Western medical acupunture: a definition. Acupunture in medicine: Journal of the British Medical Acupunture Society. 27 (1): 33-35. 2009.

27. Wynn S.G., Marsden S. Manual of natural veterinary medicine: science and tradition. Mosby Inc., St Louis, USA. 740 p. 2003.

28. Xie H., Ott E., Colahan P. Influence of acupuncture on experimental lameness in horses. Proceedings of the $47^{\text {th }}$ Annual Convention of the American Association of Equine Practitioners. 47 347-357. 2001.

29. Xie H., Preast V. Acupuncture for treatment of musculoskeletal and neurological disorders. Blackwell Publishing Ltd, Oxford, UK. p 247-265. 2007a.

30. Xie H., Preast V. Xie's veterinary acupuncture. Blackwell Publishng, lowa, USA. 2007b.

31. Yamate M. Acupuncture for the Western practitioner (Proceedings). 2009. Recuperado $23 \quad$ Febrero $2016 . \quad$ Disponible En: http://veterinarycalendar.dvm360.com/acupuncture-western-practitionerproceedings 